\title{
Correction to: What Is Hip? - Classifying Adopters and Rejecters of Interactive Digital Textiles in Home Environments
}

Julia van Heek, Philipp Brauner, and Martina Ziefle

\section{Correction to:}

Chapter "What Is Hip? - Classifying Adopters and Rejecters of Interactive Digital Textiles in Home Environments" in: C. Röcker et al. (Eds.): Information and Communication Technologies for Ageing Well and e-Health, CCIS 869, https://doi.org/10.1007/978-3-319-93644-4_1

The chapter was indvertently published with the incorrect author name information "Martina Zielfe". This has been corrected as "Martina Ziefle". 